\title{
DISCUSSÃO DE HIPÓTESES PARA MODELAGEM DA DISPERSÃO DE GASES PESADOS NA ATMOSFERA
}

César Antônio Leal

Departamento de Engenharia Nuclear, UFRGS

Av. Osvaldo Aranha, $99 / 4^{0}$ andar

90046-900 - Porto Alegre - RS - Brasil - Telefone: (051) 3163339

Edson Abel dos S. Chiaramonte

Programa de Pós-Graduação em Engenharia Mecânica, UFRGS

AV.Protásio Alves, 1187/ Apt. 02

90410-001 - Porto Alegre - RS - Brasil - Telefone: (051) 333-3170

E-mail:edsonchi@portoweb.com.br

\section{RESUMO}

As liberações de gases pesados a nível do solo na atmosfera podem ser instantânea, contínua e intermitente. Neste artigo nós apresentamos um modelo aproximado, que represente estes diversos tipos de liberações. As liberações contínua e intermitente são representadas no modelo a partir da soma e combinação de diversas liberações instantâneas sucessivas (o modelo de "puff"). Nós mostramos o modelo matemático aproximado de propriedades médias que descreve a liberação instantânea de gás pesado e as etapas do processo de soma e combinação de "puffs" para representação de uma liberação contínua (pluma). O modelo de dispersão de pluma gaussiana é usado na fase de dispersão de gás neutro. Na última 
parte do artigo, os resultados numéricos obtidos com o modelo são comparados com valores experimentais de liberações contínuas obtidos na literatura.

PALAVRAS-CHAVES: gases pesados; dispersão; excesso de densidade; pluma, entrada de ar.

\section{INTRODUÇÃO}

Busca-se entender o comportamento de liberações acidentais de gases pesados, que possuem um valor de densidade superior a do ar, a nível do solo na atmosfera, pois estes gases sendo tóxicos ou inflamáveis podem ser perigosos para pessoas, tanto nas instalações industriais como nas vizinhanças. O gás pesado possui um comportamento de dispersão na atmosfera que difere do gás leve. Estas liberações tendem a formar nuvens mais próximas ao solo, com uma largura muito maior que a sua altura (uma grande superfície de topo), conforme descrito por Fay e Zemba (1986).

As liberações de gases pesados a nível do solo na atmosfera podem ser instantânea, contínua e intermitente. Neste artigo nós apresentamos um modelo aproximado, que represente estes diversos tipos de liberações. As liberações contínua e intermitente são representadas no modelo a partir da soma e combinação de diversas liberações instantâneas sucessivas (o modelo de "puff"). Neste artigo, nós mostramos o processo de soma e combinação de "puffs" para descrever uma liberação contínua com vazão constante e os resultados numéricos obtidos com o modelo são comparados com valores experimentais obtidos na literatura.

\section{O MODELO DE LIBERAÇÃO INSTANTÂNEA}

A liberação instantânea de gás pesado é um conjunto de equações diferenciais ordinárias, que são mostradas a seguir nas equações 218 Rev. Ciência e Natura, Dispersion Process: 217 - 236 , 2000. 
(1), (2), (3), (4), (5) e (6), respectivamente, para as variáveis massa total, massa de ar incorporada, velocidade, temperatura, raio e posição do "puff". O "puff" tem a forma cilíndrica e apresenta os mesmos valores das propriedades de composição da nuvem (mistura de gás pesado e ar), temperatura e velocidade em qualquer ponto do seu interior ("box model"), conforme descrito por Wheatley (1985).

$$
\begin{aligned}
& m_{p}(t)=m_{g p}(t=0)+m_{a r}(t) \\
& \frac{d m_{p}}{d t}=\frac{d m_{a r}}{d t}=\mathrm{w}_{\mathrm{e}}(\mathrm{t}) \pi \mathrm{R}(\mathrm{t})^{2} \rho_{\text {ar }}+\mathrm{U}_{\mathrm{e}}(\mathrm{t}) 2 \pi \mathrm{R}(\mathrm{t}) \mathrm{h}(\mathrm{t}) \rho_{\text {ar }} \\
& \operatorname{com} m_{p^{(t=0)}=m_{g p}}(t=0), \text { ou m } \mathrm{ar}(\mathrm{t}=0)=0 . \\
& \frac{\mathrm{dU}}{\mathrm{dt}}=\frac{\left[\overline{\mathrm{U}}_{\mathrm{ar}}-\mathrm{U}(\mathrm{t})\right]\left(\frac{\mathrm{dm}_{\mathrm{ar}}}{\mathrm{dt}}\right)_{1}}{\left(\mathrm{~m}_{\mathrm{gp}}+\mathrm{m}_{\mathrm{ar}}\right)}+\frac{\left[\mathrm{U}_{\mathrm{ar}}-\mathrm{U}(\mathrm{t})\right]\left(\frac{\mathrm{dm}_{\mathrm{ar}}}{\mathrm{dt}}\right) \mathrm{t}}{\left(\mathrm{m}_{\mathrm{gp}}+\mathrm{m}_{\mathrm{ar}}\right)}+ \\
& +\frac{1,2 \mathrm{Rh} \rho_{\mathrm{ar}}\left[\overline{\mathrm{U}}_{\mathrm{ar}}-\mathrm{U}(\mathrm{t})\right]^{2}}{\left(\mathrm{~m}_{\mathrm{gp}}+\mathrm{m}_{\mathrm{ar}}\right)}-\frac{\left.0,0025 \pi \rho \mathrm{U}(\mathrm{t})^{2} \mathrm{R}^{2}\right\}}{\left(\mathrm{m}_{\mathrm{gp}}+\mathrm{m}_{\mathrm{ar}}\right)} \\
& {\left[\mathrm{m}_{\mathrm{ar}} \overline{\mathrm{C}}_{\mathrm{P}, \mathrm{ar}}+\mathrm{m}_{\mathrm{gp}} \overline{\mathrm{C}} \mathrm{P}, \mathrm{gp}\right] \frac{\mathrm{dT}}{\mathrm{dt}}=\frac{\mathrm{dm}_{\mathrm{ar}}}{\mathrm{dt}} \int_{\mathrm{T}}^{\mathrm{T}_{\mathrm{ar}}} \overline{\mathrm{C}}_{\mathrm{P}, \mathrm{ar}} \mathrm{dT}+} \\
& +\frac{\mathrm{C}_{\mathrm{f}}}{2} \mathrm{U} \rho \overline{\mathrm{C}} \mathrm{P}, \mathrm{p}\left(\pi \mathrm{R}^{2}\right)\left(\mathrm{T}_{\mathrm{ar}}-\mathrm{T}\right) \\
& \frac{d R}{d t}=1,3 \sqrt{g h \frac{\left(\rho-\rho_{a r}\right)}{\rho}} \\
& \frac{\mathrm{dX}_{\mathrm{C}}}{\mathrm{dt}}=\mathrm{U}
\end{aligned}
$$

onde:

$\mathrm{m}_{\mathrm{p}}(\mathrm{t})=$ massa do "puff" (gás pesado mais ar) no instante de tempo t, em kg. 
$\mathrm{m}_{\mathrm{ar}}(\mathrm{t})=$ massa de ar no interior do "puff", em kg.

$\mathrm{m}_{\mathrm{gp}}(\mathrm{t}=0)=$ massa do "puff" de gás pesado liberado na fonte, em $\mathrm{kg}$.

$t=$ tempo total do "puff" desde a sua liberação, em s.

$W_{e}(t)=$ velocidade de entrada de ar no topo do "puff", no instante de tempo $t$, em $\mathrm{m} / \mathrm{s}$.

$R(t)=$ raio do "puff", no instante $t$, em $m$.

$\rho_{a r}=$ densidade do $\mathrm{ar}$, em $\mathrm{kg} / \mathrm{m}^{3}$.

$\mathrm{U}_{\mathrm{e}}(\mathrm{t})=$ velocidade de entrada de ar pela superfície lateral do "puff", em m/s.

$h(t)=$ altura do "puff", no instante $t$, em $\mathrm{m}$.

$\bar{U}_{\text {ar }}=$ velocidade média do vento calculada na altura do "puff", em m/s.

$\mathrm{U}_{\mathrm{ar}}=$ velocidade do vento na altura do topo do "puff", em m/s.

$\mathrm{U}(\mathrm{t})=$ velocidade do "puff", em $\mathrm{m} \mathrm{s}^{-1}$.

$\left(\frac{\mathrm{dm}_{\mathrm{ar}}}{\mathrm{dt}}\right)_{1}=$ entrada de ar pela superfície lateral do "puff", no instante t, em kg $\mathrm{s}^{-1}$.

$\left(\frac{\mathrm{dm}_{\mathrm{ar}}}{\mathrm{dt}}\right)_{\mathrm{t}}=$ entrada de ar pela superfície de topo do "puff", no instante t, em kg $\mathrm{s}^{-1}$.

$\mathrm{T}=$ temperatura do "puff" de gás pesado, em $\mathrm{K}$.

$\mathrm{CP}_{\mathrm{g} g \mathrm{~g}}=$ calor específico a pressão constante para a mistura de gás pesado no interior do "puff", em J kg-1 $\mathrm{K}^{-1}$.

$\overline{\mathrm{C}} \mathrm{P}, \mathrm{ar}=$ calor específico a pressão constante para a massa de ar no interior do "puff", $\mathrm{J} \mathrm{kg}^{-1} \mathrm{~K}^{-1}$.

$\overline{\mathrm{C}} \mathrm{P}, \mathrm{p}=$ calor específico a pressão constante para o "puff" de gás pesado, em $\mathrm{J} \mathrm{kg}^{-1} \mathrm{~K}^{-1}$.

$X_{C}=$ Posição do centro do "puff" em relação a fonte de liberação, em m.

220 Rev. Ciência e Natura, Dispersion Process: 217 - 236 , 2000. 
As equações diferenciais ( 2 ), ( 3 ), ( 4 ), ( 5 ), e ( 6 ) são resolvidas pelo método de Runge-Kutta de $4^{\text {a }}$ ordem. As densidades da nuvem de gás pesado e do ar são determinadas por uma equação de estado. O modelo de liberação instantânea pode ser visto em detalhe nos trabalhos de Leal e Chiaramonte (1998) e Eidsvik (1980).

\section{O MÉTODO DE SOMA DE "PUFFS" PARA REPRESENTAR FONTES CONTÍNUAS DE DISPERSÃO DE GASES PESADOS}

Uma liberação de gás pesado pode ser considerada instantânea, ou pode ter um intervalo grande de tempo para deixar a fonte. A liberação com intervalo grande de duração pode possuir diversos comportamentos, tais como a vazão ser contínua e constante, ou contínua e decrescer exponencialmente com o tempo de liberação, ou uma fonte intermitente. O objetivo desta seção é apresentar um método de representação destas diversas formas de liberações efetuada a partir da soma de diversas liberações instantâneas independentes ("puffs"). O modelo, ainda em desenvolvimento, para representar uma fonte contínua com vazão constante será descrito a seguir.

O modelo da pluma contínua de gás pesado com vazão constante é composto por dois modelos de dispersão atmosférica. O primeiro representa a pluma de gás pesado, que é formada pela soma de "puffs", quando a dispersão lateral é governada pelo seu excesso de densidade. $O$ segundo é o modelo de pluma gaussiana, quando o regime de dispersão é dominado pela turbulência atmosférica (a pluma não possui mais o excesso de densidade). $O$ modelo faz esta passagem da pluma de gás pesado para o modelo de dispersão de gás leve (pluma gaussiana).

O sistema que representa a pluma de gás pesado por uma soma de um conjunto de "puffs", mostrado no esquema das Figuras 1 e 2 , tem os seguinte procedimentos:

Rev. Ciência e Natura, Dispersion Process: 217 - 236 , 2000. 


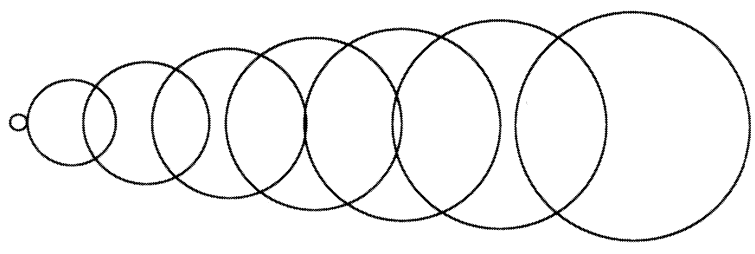

FONTE

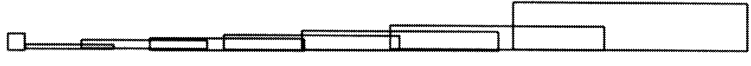

\begin{tabular}{l|l|l|l|l|l|l}
$10 \mathrm{~m}$ & $100 \mathrm{~m}$ & $200 \mathrm{~m}$ & $500 \mathrm{~m}$ & $400 \mathrm{~m}$ & $500 \mathrm{~m}$ & $600 \mathrm{~m}$
\end{tabular}

Figura 1: Esquema de formação da pluma de gás pesado através da soma de "puffs".

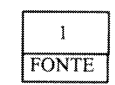

(a) Liberação do "puff número 1.

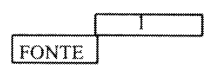

(d) Teste para ver se o "puff" é gás leve.

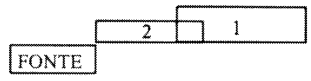

(g) Teste para ver se os "puffs" são gás leve.

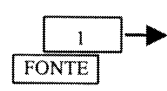

(b) Movimentação do "puff" número 1.

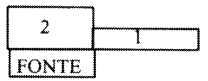
(e) Liberação do "puff
número 2 .

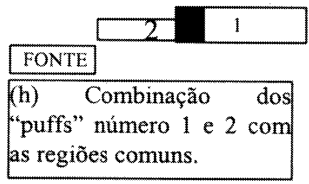

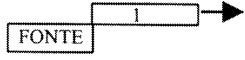

(c) Movimentação do "puff" número 1.

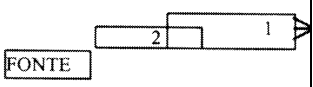

(f) Movimentação dos "puffs" números 1 e 2.

\begin{tabular}{|l|l|}
\hline 3 & 251 \\
\hline FONTE & 1 \\
\hline
\end{tabular}

(i) Liberação do "puff número 3.

Figura 2: Sistema de liberação e combinação de "puffs" de gás pesado.

222 Rev. Ciência e Natura, Dispersion Process: 217 - 236 , 2000. 
a) Modelo de liberação de "puffs" independentes sobre a fonte

Cada "puff" de gás pesado tem um tempo de formação sobre a fonte de liberação, conforme mostrado na Figura 3. Para a liberação contínua com vazão constante foi adotado o mesmo tempo de formação para todos os "puffs".

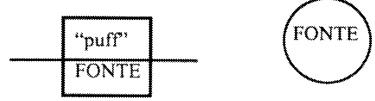

( a ) O 'puff' sobre a fonte após o tempo de liberação $\left(\mathrm{t}=\mathrm{t}_{\mathrm{f}}\right)$.

[FONTE "puff"

( c ) O "puff" não deixa a fonte após o de tempo de liberação $\left(t=2 t_{\text {pUFF }}\right)$.
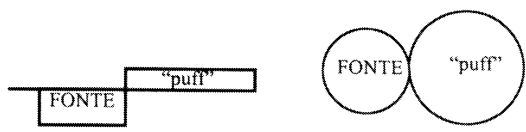

( b ) O 'puff' deixa a fonte após o de tempo de liberação $\left(t=2 t_{\text {PUEF }}\right)$.

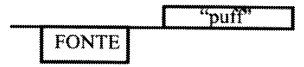

( d ) O 'puff" ultrapassa a fonte após o de tempo de liberação( $\left(t=2 t_{\text {purE }}\right)$.

Figura 3: O tempo de liberação do "puff" na fonte.

b) Sistema de soma e combinação dos "puffs" de gás pesado é constituído das seguintes etapas:

b.1) Escoamento e dispersão dos "puffs" de gás pesado

O conjunto de "puffs" de gás pesado da pluma são movimentados a cada período de tempo de formação de um novo "puff" e cada um independentemente segue o modelo de dispersão descrito na seção 2 .

b.2) Processo de combinação dos "puffs" de gás pesado

Os "puffs" que formam a pluma apresentam regiões comuns entre si, conforme mostrado na Figura 1. Após cada período em que são movimentados, os "puffs" passam por procedimentos de combinação de suas propriedades independentes. As propriedades são a composição da mistura, velocidade e temperatura nestas regiões. 
A mistura perfeita nas regiões comuns foi o processo de combinação adotado e é realizado no final de cada período de movimentação da pluma. O processo adota o equilíbrio de massa, energia e quantidade de movimento nas regiões. Um "puff" da pluma é combinado com os "puffs" que estão localizados adjacentes a ele nas posições anterior e posterior.

O processo de equilíbrio do balanço de massa:

A massa total do "puff" de índice 1 na região comum e a massa total na região comum são:

$$
\mathrm{m}_{\mathrm{p}, 1}^{\mathrm{C}}=\mathrm{m}_{\mathrm{gp}, 1}+\mathrm{m}_{\mathrm{ar}, 1}
$$

onde:

$\mathrm{m}_{\mathrm{p}, 1}^{\mathrm{C}}=$ massa total do "puff" número 1 na região comum, em kg.

$\mathrm{m}_{\mathrm{gp}, 1}=$ massa de gás pesado do "puff" número 1 na região comum, em kg.

$\mathrm{m}_{\mathrm{ar}, 1}=$ massa de ar do "puff" número 1 na região comum, em kg.

$$
\mathrm{m}_{\mathrm{t}}^{\mathrm{C}}=\mathrm{m}_{\mathrm{gp}, 1}+\mathrm{m}_{\mathrm{ar}, 1}+\mathrm{m}_{\mathrm{gp}, 2}+\mathrm{m}_{\mathrm{ar}, 2}
$$

onde:

$\mathrm{m}_{\mathrm{t}}^{\mathrm{C}}=$ massa total na região comum, em $\mathrm{kg}$.

$\mathrm{m}_{\mathrm{gp}, 2}=$ massa de gás pesado do "puff" número 2 na região comum, em kg.

$\mathrm{m}_{\mathrm{ar}, 2}=$ massa de ar do "puff" número 2 na região comum, em kg.

A massa de gás pesado do "puff" número 1, após o processo de mistura perfeita, é calculada considerando que o "puff" conserva a mesma quantidade de massa e a composição da mistura.

$$
\mathrm{m}_{\mathrm{gp}, 1}=\frac{\mathrm{m}_{\mathrm{p}, 1}}{\mathrm{~m}_{\mathrm{t}}^{\mathrm{C}}}\left(\mathrm{m}_{\mathrm{gp}, 1}+\mathrm{m}_{\mathrm{gp}, 2}\right)
$$

224 Rev. Ciência e Natura, Dispersion Process: 217 - 236 , 2000. 
Este processo de mistura perfeita de massa nas regiões comuns entre os "puffs" mostrou transferir uma quantidade de massa elevada do "puff" mais diluído para o mais concentrado. Esta quantidade de massa transferida é limitada por um modelo de difusão aproximado.

O processo de equilíbrio do balanço de energia na região comum, que determina a temperatura de equilíbrio é:

$\int_{\mathrm{T}_{1}}^{\mathrm{T}} \mathrm{m}_{\mathrm{gp}, 1} \mathrm{C}_{\mathrm{P}, \mathrm{gp}} \mathrm{dT}+\int_{\mathrm{T}_{1}}^{\mathrm{T}} \mathrm{m}_{\mathrm{ar}, 1}^{\mathrm{C}} \mathrm{C}_{\mathrm{P}, \mathrm{ar}} \mathrm{dT}+\int_{\mathrm{T}_{2}}^{\mathrm{T}} \mathrm{m}_{\mathrm{gp}, 2} \mathrm{C}_{\mathrm{P}, \mathrm{gp}} \mathrm{dT}+\int_{\mathrm{T}_{2}}^{\mathrm{T}} \mathrm{m}_{\mathrm{ar}, 2} \mathrm{C}_{\mathrm{P}, \mathrm{ar}} \mathrm{dT}=0$

onde:

$\mathrm{C}_{\mathrm{P}, \mathrm{gp}}=$ calor específico do gás ideal à pressão constante para a mistura de gás pesado, em $\mathrm{J} \mathrm{kg}^{-1} \mathrm{~K}^{-1}$.

$\mathrm{C}_{\mathrm{P}, \mathrm{ar}}=$ calor específico do gás ideal à pressão constante para $\mathrm{o}$ ar atmosférico, em $\mathrm{J} \mathrm{kg}^{-1} \mathrm{~K}^{-1}$.

$\mathrm{T}_{1}=$ temperatura do "puff" número "1", antes do processo de mistura, em $\mathrm{K}$.

$\mathrm{T}_{2}=$ temperatura do "puff" número "2", antes do processo de mistura, em $\mathrm{K}$.

$\mathrm{T}^{\mathrm{C}}=$ temperatura de equilíbrio na região comum entre os "puffs" de números "1" e "2", em K.

$O$ processo de equilíbrio do balanço de quantidade de movimento na região comum, que determina a velocidade de equilíbrio é:

$$
\mathrm{U}^{\mathrm{C}}=\frac{\mathrm{U}_{1} \mathrm{~m}_{\mathrm{p}, 1}^{\mathrm{C}}+\mathrm{U}_{2} \mathrm{~m}_{\mathrm{p}, 2}^{\mathrm{C}}}{\mathrm{m}_{\mathrm{p}, 1}^{\mathrm{C}}+\mathrm{m}_{\mathrm{p}, 2}^{\mathrm{C}}}
$$

onde:

$\mathrm{U}^{\mathrm{C}}=$ velocidade de equilíbrio na região comum dos "puffs" de números "1" e "2", em m s"-1.

$\bigcup_{1}=$ velocidade do "puff" número "1", antes do processo de mistura, em $\mathrm{m} \mathrm{s}^{-1}$. $\mathrm{U}_{2}=$ velocidade do "puff" número "2", antes do processo de mistura, em m s s. $^{-1}$. 
$\mathrm{m}_{\mathrm{p}, 2}^{\mathrm{C}}=$ massa total do "puff" número 2 na região comum, em kg.

O sistema de combinação também diminui a área de entrada de ar para cada "puff" cilíndrico devido as regiões comuns e testa se o "puff" mais diluído passou para a condição de dispersão de gás neutro, usando os critérios empíricos adotados por Fryer e Kayser (1978) e Melhen e Little (1992).

\section{A TROCA PARA O MODELO DE DISPERSÃO DE GÁS LEVE}

A pluma contínua segue o modelo de dispersão de pluma gaussiana, quando o seu regime de dispersão for dominado pela turbulência atmosférica. A concentração da pluma é obtida pela relação

$$
\rho(\mathrm{x}, \mathrm{y}, \mathrm{z})=\frac{\mathrm{Q}}{2 \pi \mathrm{u} \sigma_{\mathrm{y}} \sigma_{\mathrm{z}}} \exp \left(-\frac{1}{2}\left(\frac{\mathrm{y}^{2}}{\sigma_{\mathrm{y}}^{2}}+\frac{\mathrm{z}^{2}}{\sigma_{\mathrm{z}}^{2}}\right)\right)
$$

onde:

$\rho(x, y, z)=$ concentração da pluma no ponto de coordenadas $(x, y, z), \mathrm{em} \mathrm{kg} \mathrm{m}^{-3}$.

$\mathrm{Q}=$ vazão mássica da fonte, $\mathrm{em} \mathrm{kg} \mathrm{s}^{-1}$.

$\overline{\mathrm{u}}=$ velocidade média do vento, em $\mathrm{m} \mathrm{s}^{-1}$.

$\sigma_{y}=$ coeficiente de dispersão atmosférica horizontal, em $\mathrm{m}$.

$\sigma_{\mathrm{z}}=$ coeficiente de dispersão atmosférica vertical, em $\mathrm{m}$.

$\mathrm{x}=$ distância a fonte de emissão do gás (virtual), em $\mathrm{m}$.

$y=$ distância horizontal do centro da pluma, em $\mathrm{m}$.

$\mathrm{z}=$ distância vertical do solo, em $\mathrm{m}$.

Os coeficientes de dispersão atmosférica nas direções vertical e horizontal são determinados por relações empíricas, mostradas por van Buijtenen (1979):

226 Rev. Ciência e Natura, Dispersion Process: 217 - 236 , 2000. 


$$
\begin{aligned}
& \sigma_{y}\left(\mathrm{x}_{\mathrm{vy}}\right)=\mathrm{ax}_{\mathrm{vy}}^{\mathrm{b}} \\
& \sigma_{z}\left(\mathrm{x}_{\mathrm{vz}}\right)=\mathrm{c}\left(10 \mathrm{Z}_{0}\right)^{0,53 \cdot \mathrm{x}_{\mathrm{VZ}}^{-0,22} \mathrm{x}_{\mathrm{vz}}^{\mathrm{d}}}
\end{aligned}
$$

onde:

Xvy = distância virtual de uma fonte pontual na direção horizontal, em $\mathrm{m}$,

$\mathrm{x}_{\mathrm{vz}}=$ distância virtual de uma fonte pontual na direção vertical, em $\mathrm{m}$,

$Z_{0}=$ comprimento de rugosidade do solo, em $\mathrm{m} \mathrm{e}$

a, b, c e d = são parâmetros determinados em função das classes de estabilidade atmosférica de Pasquill-Gifford.

Após a passagem para o modelo de pluma gaussiana, a concentração passa a ser avaliada considerando-se "puffs" cilíndricos aproximados de dimensões de raio e altura determinados pelas relações ( 18 ) e ( 19 ), que serão mostradas a seguir. Nestes a concentração máxima é obtida com a equação ( 12 ) sobre o eixo da pluma ao nível do solo (com as distancias horizontal e vertical com valores zero), e a concentração média obtida com a relação:

$$
\bar{\rho}_{\mathrm{plu}}=\frac{\operatorname{Qerf}\left(\frac{\mathrm{H}}{\sqrt{2} \sigma_{\mathrm{z}}}\right) \operatorname{erf}\left(\frac{\mathrm{R}}{\sqrt{2} \sigma_{\mathrm{y}}}\right)}{2 \overline{\mathrm{uRH}}}
$$

onde:

$\bar{\rho}_{\text {plu }}=$ concentração média do perfil de pluma gaussiana em uma região cilíndrica, em $\mathrm{kg} \mathrm{m}^{-3}$,

$\mathrm{R}=$ raio do "puff" cilindro, em $\mathrm{m}$.

$\mathrm{H}=$ altura do "puff" cilindro, em $\mathrm{m}$.

$\operatorname{erf}(x)=$ função erro do argumento $x$. 


\section{A troca do modelo de dispersão de pluma de gás pesado para gás leve}

Os modelos de dispersão de gases pesados determinam as coordenadas de fonte virtual nas direções vertical e horizontal, no instante de transição do modelo de dispersão, a partir das relações a seguir:

$$
\begin{aligned}
& \mathrm{R}_{\mathrm{t}}=2,15 \sigma_{\mathrm{yt}} \\
& \frac{\mathrm{H}_{\mathrm{t}}}{2}=2,15 \sigma_{\mathrm{zt}}
\end{aligned}
$$

onde:

$\mathrm{R}_{\mathrm{t}}=$ raio do "puff" no instante da transição, em $\mathrm{m}$.

$\mathrm{H}_{\mathrm{t}}=$ altura do "puff" no instante da transição, em m.

$\sigma_{y t}=$ coeficiente de dispersão atmosférica horizontal no instante da transição, em $\mathrm{m}$.

$\sigma_{\mathrm{z}} \mathrm{t}=$ coeficiente de dispersão atmosférica vertical no instante da transição, em $\mathrm{m}$.

Neste trabalho, introduziu-se um método para determinar o valor do coeficiente de proporcionalidade entre as dimensões do "puff" de gás pesado e os coeficientes de dispersão para que o modelo de pluma gaussiana contínua mantenha o valor de concentração média, no instante da transição.

Há uma proporcionalidade entre as dimensões do "puff" e os coeficientes de dispersão:

$$
\begin{aligned}
& \mathrm{R}_{\mathrm{t}}=\mathrm{C}_{\mathrm{t}} \sigma_{\mathrm{yt}} \\
& \frac{\mathrm{H}_{\mathrm{t}}}{2}=\mathrm{C}_{\mathrm{t}} \sigma_{\mathrm{zt}}
\end{aligned}
$$

onde:

$\mathrm{C}_{\mathrm{t}}=$ coeficiente de proporcionalidade para $\mathrm{o}$ instante da transição de gás pesado para leve.

228 Rev. Ciência e Natura, Dispersion Process: 217 - 236 , 2000. 
O coeficiente de proporcionalidade é determinado pelo valor que satisfaz a equação a seguir.

$$
\frac{\mathrm{Q}_{\mathrm{gp}}\left[\operatorname{erf}\left(\frac{\mathrm{C}_{\mathrm{t}}}{\sqrt{2}}\right)\right]^{2}}{2 \mathrm{U}_{\mathrm{t}} \mathrm{R}_{\mathrm{t}} \mathrm{H}_{\mathrm{t}}}-\frac{\mathrm{m}_{\mathrm{gp}, \mathrm{t}}}{\left(\mathrm{m}_{\mathrm{gp}, \mathrm{t}}+\mathrm{m}_{\mathrm{ar}, \mathrm{t}}\right)} \rho_{\mathrm{p}, \mathrm{t}}=0
$$

onde:

$\rho_{\mathrm{gp}, \mathrm{t}}=$ concentração de gás pesado do "puff", no instante da transição para gás leve, em kg m${ }^{-3}$,

$m_{\mathrm{gp}, \mathrm{t}}=$ massa de gás pesado do "puff", no instante da transição para gás leve, em kg,

$m_{a r, t}=$ massa de ar do "puff", no instante da transição para gás leve, em kg e $\rho_{p, t}=$ concentração total do "puff", no instante da transição para gás leve, em $\mathrm{kg} \mathrm{m}^{-3}$.

$U_{t}=$ velocidade do "puff" de gás pesado, no instante de transição para gás leve, em $\mathrm{m} \mathrm{s}^{-1}$.

\section{RESULTADOS OBTIDOS E COMPARAÇÃO COM DADOS EXPERIMENTAIS}

Os resultados obtidos com o modelo são comparados aos testes de campo de Maplin Sands conduzidos pela Shell Oil Company, que são liberações contínuas de GNL (Gás Natural Liquefeito) e GLP (Gás Liquefeito de Petróleo) sobre água, conforme mostrados por Havens (1992). Para essas liberações foram obtidas as concentrações de gás pesado a várias distâncias medidas a partir do centro da fonte de emissão, onde a concentração é representada pela porcentagem do número de moles da mistura de gás pesado liberada na fonte.

Na figura 4 são apresentados os gráficos da concentração contra a distância do centro da fonte para os testes de Maplin 27 e 54, 
respectivamente. Os resultados experimentais de campo são representados nos gráficos pelos pontos em asterisco e fornecem a porcentagem máxima do número de moles da mistura de gás pesado a várias distâncias. Os resultados obtidos com o modelo de "puffs" são representados nos gráficos pela linha contínua, onde os pontos na linha contínua representam a porcentagem do número de moles na distância do centro de cada um dos "puffs" que formam a pluma.

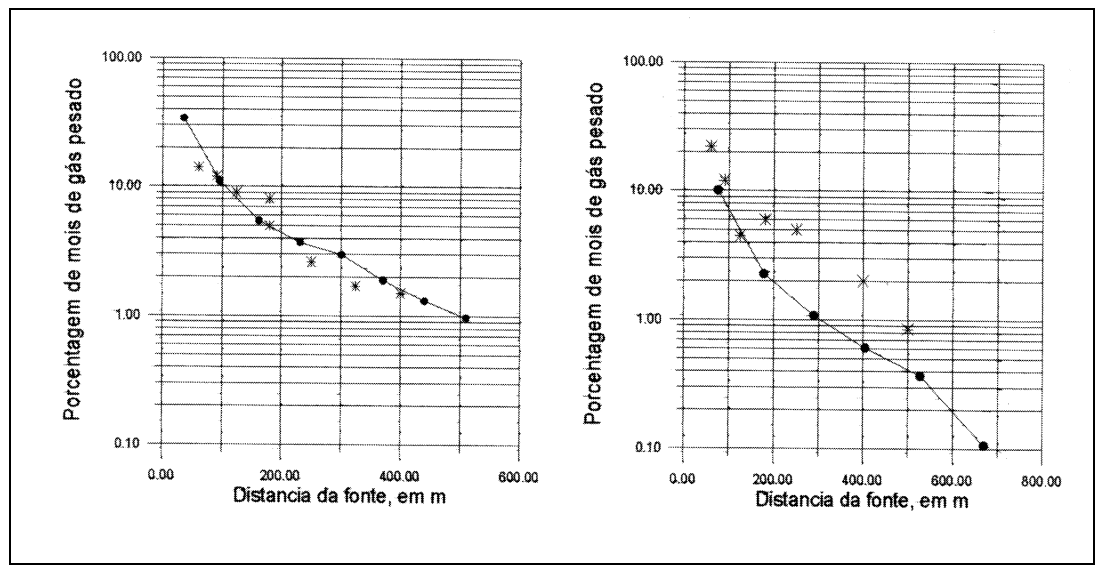

Figura 4: Gráficos da porcentagem da mistura de gás pesado liberada na fonte contra a distancia ao centro da fonte para os testes de Maplin 27 e 54.

Nos gráficos das figuras 5 e 7 mostra-se os resultados do modelo (linha contínua) comparados com os resultados, quando o modelo que adota a velocidade de deslocamento dos "puffs" igual a velocidade média do vento na sua altura (o modelo adotado pelos "box models" preexistentes), desde o instante de sua liberação na fonte (linha tracejada). No modelo de velocidade média, os "puffs" abandonam a fonte mais rápido, com um tempo de formação menor. Enquanto os gráficos das figuras 6 e 8 mostram a

230 Rev. Ciência e Natura, Dispersion Process: 217 - 236 , 2000. 
influencia do termo de arraste na equação de balanço de quantidade de movimento aproximada, onde a linha pontilhada representa os resultados sem a presença desse termo (valor do coeficiente de arraste igual a zero), e nesse modelo os "puffs" tem um tempo de formação maior na fonte .Nas figuras 5 a 8 , também são mostrados os desenhos das vistas superior $\mathrm{e}$ lateral do conjunto de "puffs", obtidos com o modelo, que formam a pluma de gás pesado. A escala que foi utilizada é de 1:60 e para a altura foi usada uma escala de redução menor de 1:5, porque os valores das alturas são pequenos em relação aos raios dos "puffs". O desenho a esquerda é o resultado para o modelo apresentado, enquanto o da direita representa o resultado com o modelo de velocidade média. Os desenhos mostram a influência do modelo de velocidade do "puff" na dispersão lateral da pluma de gás pesado e no números de "puffs" para forma-la. 


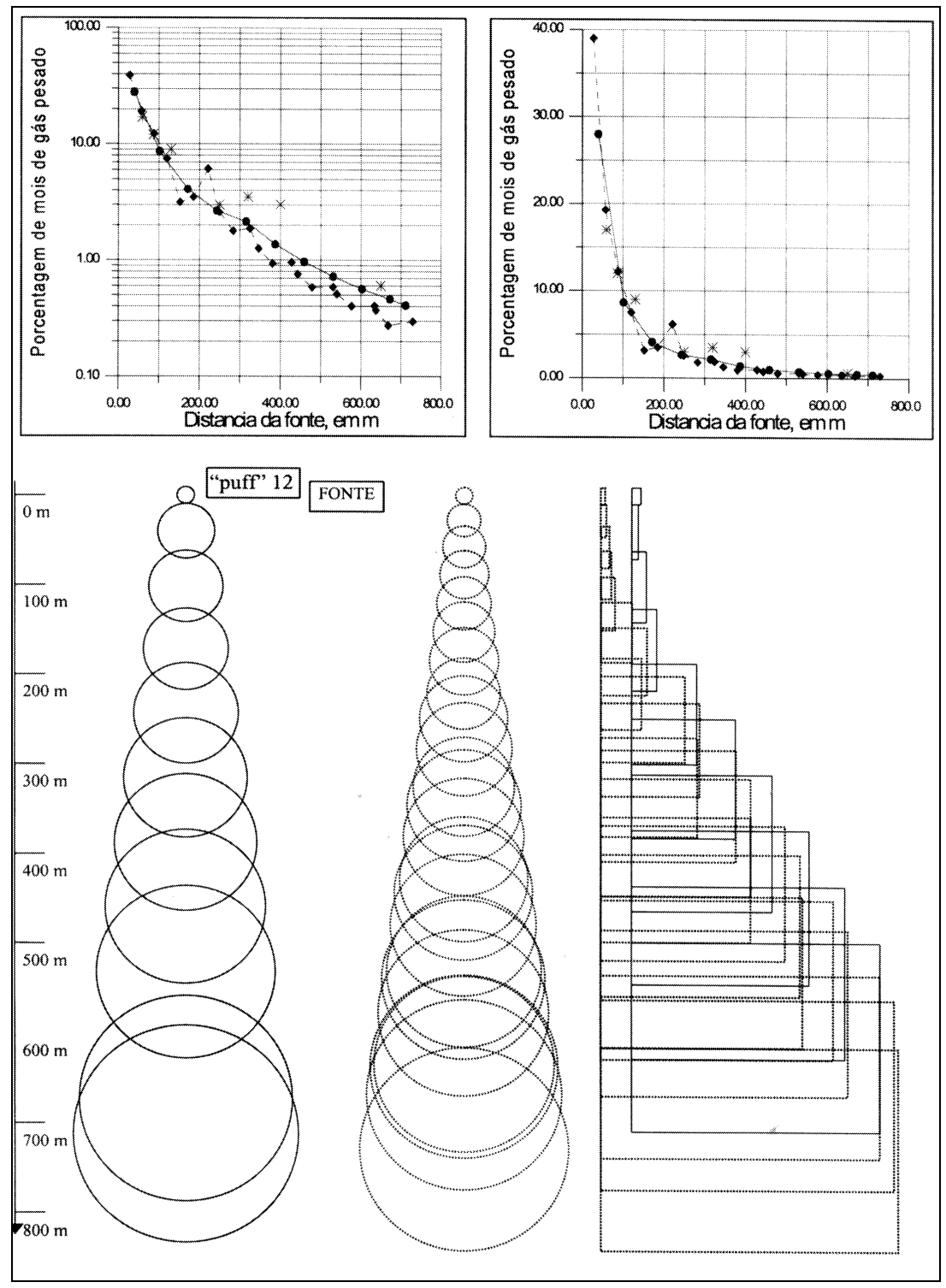

Figura 5: Comparação da velocidade inicial do "puff" para o teste Maplin 27.

232 Rev. Ciência e Natura, Dispersion Process: 217 - 236 , 2000. 


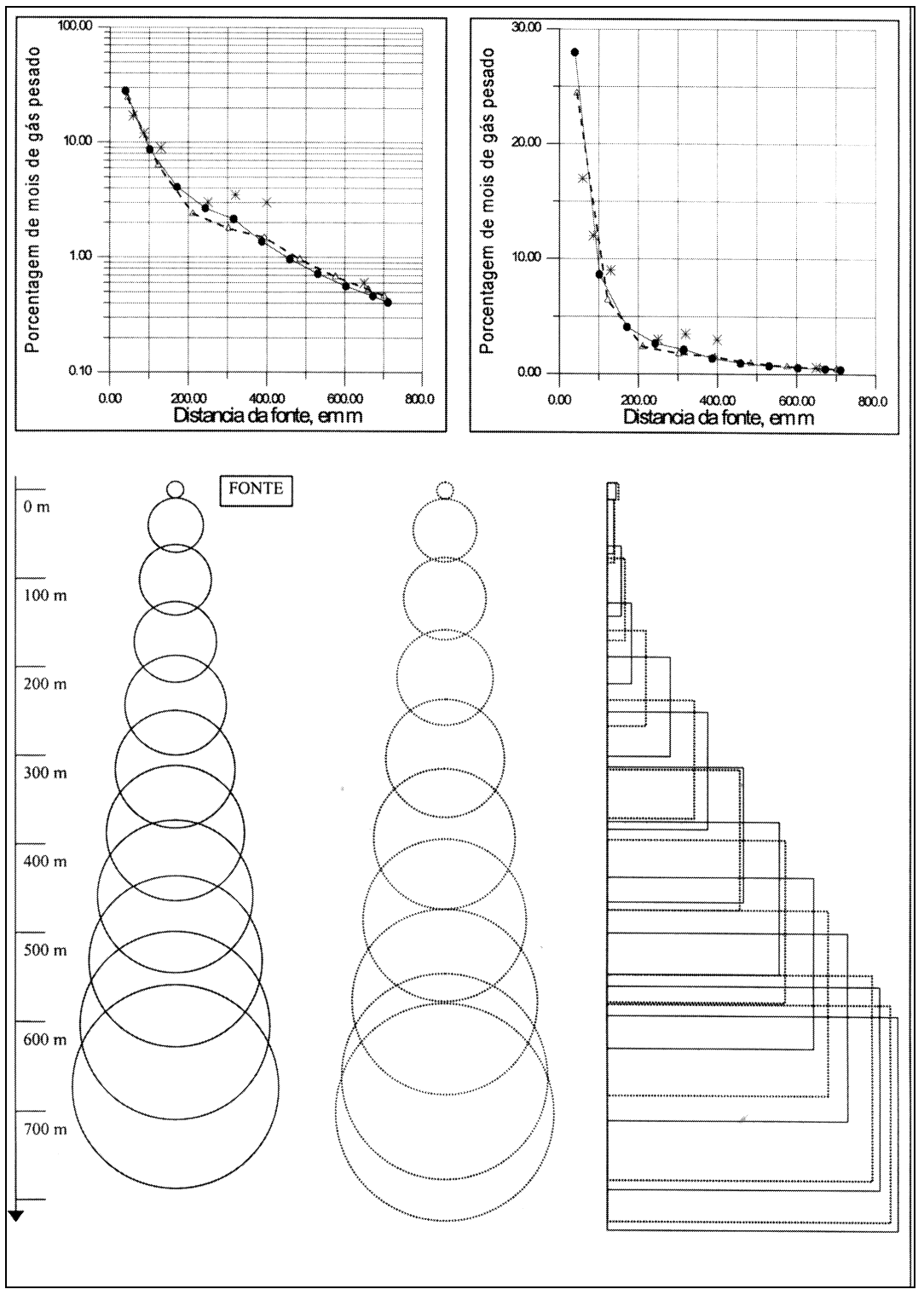

Figura 6: Comparação do valor do coeficiente de arraste para o teste

Maplin 27.

Rev. Ciência e Natura, Dispersion Process: 217 - 236 , 2000. 

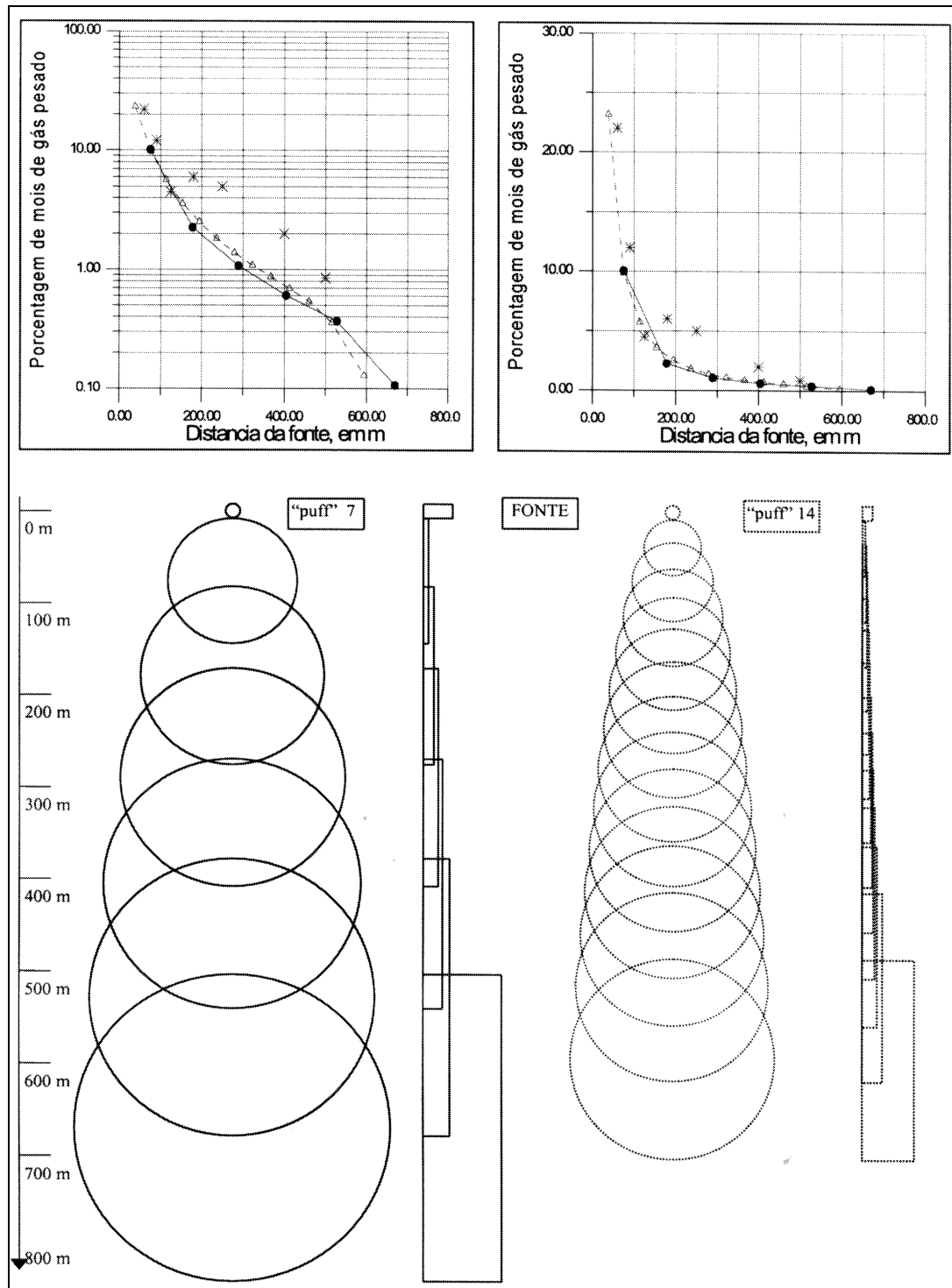

Figura 7: Comparação da velocidade inicial ar para o teste Maplin 54.

234 Rev. Ciência e Natura, Dispersion Process: 217 - 236 , 2000. 

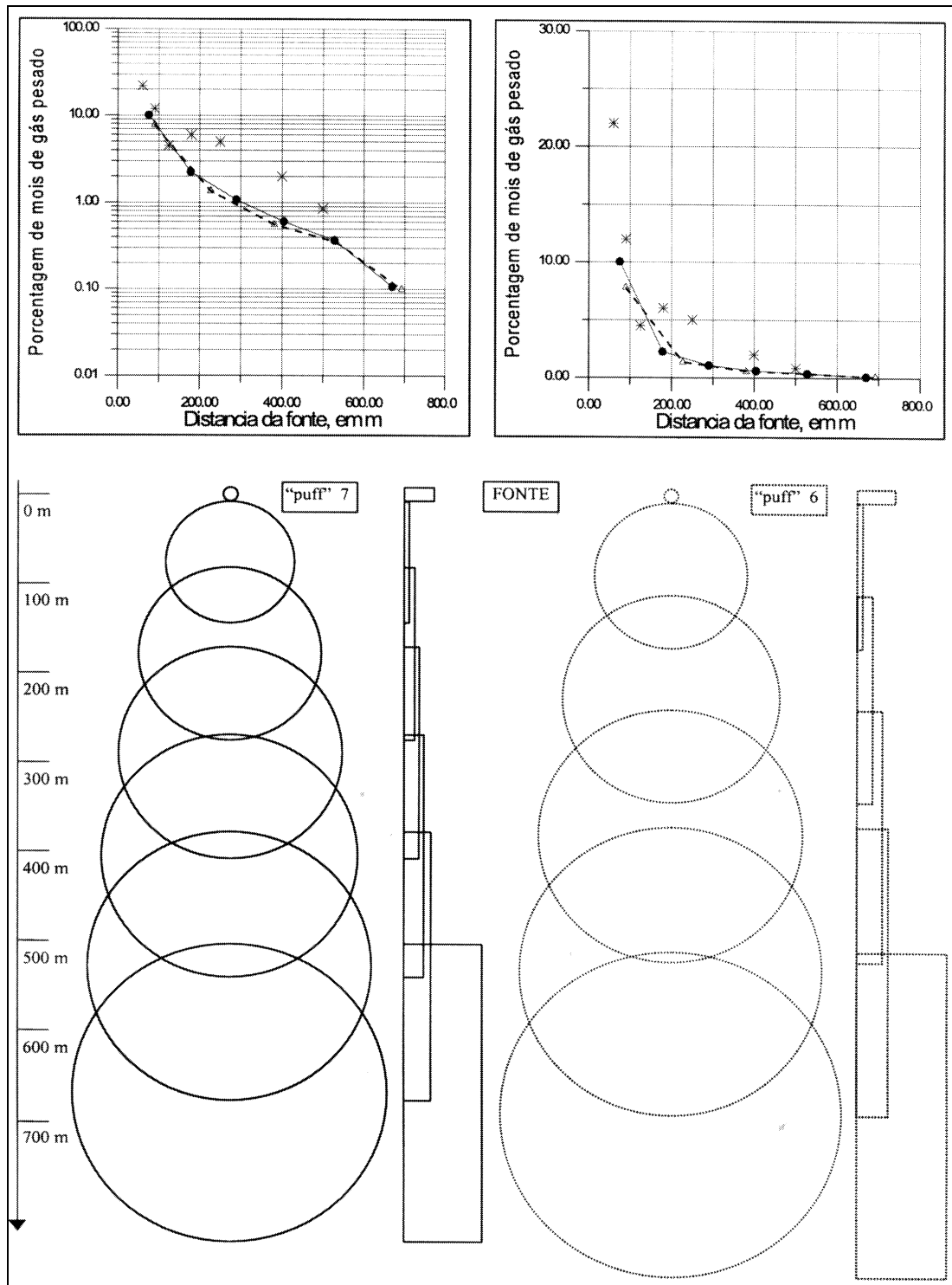

Figura 8: Comparação do valor do coeficiente de arraste para o teste Maplin 54.

Rev. Ciência e Natura, Dispersion Process: 217 - 236 , 2000. 


\section{BIBLIOGRAFIA}

[1] Eidsvik, K. J., 1980. "A Model for Heavy Gas Dispersion in the Atmosphere", Atmospheric Environment, Vol. 14, pp. 769-777.

[2] Fay, J. A. e S. G. Zemba, 1986. "Integral Model of Dense Gas Plume Dispersion", Atmospheric Environment, Vol. 20, pp. 1347-1354.

[3] Fryer, L. S. e G. D. Kaiser, 1979. DENZ - A computer program for the calculation of the dispersion of dense toxic or explosive gases in the atmosphere, Technical Report SRD-R152, Safety and Reliability Directorate, Inglaterra.

[4] Havens, J., 1992. "Review of dense gas dispersion field experiments", J. Loss Prev. Process Ind.,Vol. 5, pp. 28-41.

[5] Leal, C. e Chiaramonte, E., 1998. "Simulação numérica do comportamento de "puffs" para representar a dispersão de gases pesados na atmosfera", $12^{\circ}$ Congresso Brasileiro de Engenharia Química, Porto Alegre.

[6] Melhem G. A. e A. D. Little, 1992. Advanced Consequence Modeling Emission, Dispersion, Fires and Explosions, Van Nostrand Reinhold, New York.

[7] van Buijtenen, C. J. P., 1979. Methods for the calculation of the physical effects of the escape of dangerous material (liquids and gases) Part II ${ }_{2}$ DISPERSION, Chapter 7, Directorate-General of Labour, Netherlands. [8] Wheatley, C. J., 1985. Dispersion of Heavy Gases, Von Karman Institute for Fluid Dynamics. 